\title{
Area evolution, bulk viscosity and entropy principles for dynamical horizons
}

\author{
Eric Gourgoulhor* and José Luis Jaramilld \\ Laboratoire de l'Univers et de ses Théories, UMR 8102 du C.N.R.S., \\ Observatoire de Paris, F-92195 Meudon Cedex, France
}

(Dated: 6 September 2006)

\begin{abstract}
We derive from Einstein equation an evolution law for the area of a trapping or dynamical horizon. The solutions to this differential equation show a causal behavior. Moreover, in a viscous fluid analogy, the equation can be interpreted as an energy balance law, yielding to a positive bulk viscosity. These two features contrast with the event horizon case, where the non-causal evolution of the area and the negative bulk viscosity require teleological boundary conditions. This reflects the local character of trapping horizons as opposed to event horizons. Interpreting the area as the entropy, we propose to use an area/entropy evolution principle to select a unique dynamical horizon and time slicing in the Cauchy evolution of an initial marginally trapped surface.

PACS numbers: 04.70.Bw, 04.20.-q, 04.70.-s
\end{abstract}

Introduction. A new paradigm has recently emerged in the theoretical approaches to black holes, following the introduction of future outer trapping horizons (FOTH) by Hayward [1, 2] and that of dynamical horizons (DH) by Ashtekar and Krishnan 3] (see Refs. [4, 5] for reviews). This new approach relies on local characterizations of black holes, via trapped surfaces, and contrasts with the traditional treatment which is based on the notion of event horizon $(E H)$. The latter is a highly non-local concept and requires the knowledge of the whole spacetime to be determined. This feature makes the EH a not very practical representation of black holes for studies in numerical relativity and quantum gravity, and motivated the new approach.

In this note, we focus on the evolution of the area of cross sections of FOTHs and DHs. We obtain an area law of different nature from the "area increase law" derived by Ashtekar and Krishnan [3, 4]. Inspired by the membrane paradigm developed for EHs [6, 7], we treat the cross sections of FOTHs and DHs as viscous fluid bubbles and interpret the area law as an internal energy balance equation, completing the Navier-Stokes-like momentum law derived previously [8]. It is then apparent that the bulk viscosity is positive. Associating area with entropy, we also discuss the use of an entropy principle to pick up a unique dynamical horizon in an evolution. Both a maximum entropy production and a time convexity requirement (interpretable in terms of ClausiusDuhem inequality) are considered.

Evolution of the horizon area. Both the $\mathrm{EH}$ and the FOTH/DH approaches to black holes can be formulated in terms of a hypersurface $\mathcal{H}$ embedded in a fourdimensional spacetime $(\mathcal{M}, \boldsymbol{g})$, and foliated by a family of closed (topologically $\mathbb{S}^{2}$ ) spacelike 2 -surfaces $\left(\mathcal{S}_{t}\right)_{t \in \mathbb{R}}$. If it represents an $\mathrm{EH}, \mathcal{H}$ is a null hypersurface and any foliation $\left(\mathcal{S}_{t}\right)_{t \in \mathbb{R}}$ is admissible. In the case of a $\mathrm{DH}, \mathcal{H}$ is a

*eric.gourgoulhon@obspm.fr

†jose-luis.jaramillo@obspm.fr spacelike hypersurface and the foliation $\left(\mathcal{S}_{t}\right)_{t \in \mathbb{R}}$ is unique [ $t$ defined up to a relabeling $\left.t \mapsto t^{\prime}=f(t)\right]$, each $\mathcal{S}_{t}$ being a marginally trapped surface [9].

Given the foliation $\left(\mathcal{S}_{t}\right)_{t \in \mathbb{R}}$ of $\mathcal{H}$, there is a unique "time evolution" vector $\boldsymbol{h}$ that is tangent to $\mathcal{H}$, orthogonal to $\mathcal{S}_{t}$ and obeys $\mathcal{L}_{\boldsymbol{h}} t=1$, where $\mathcal{L}_{\boldsymbol{h}}$ denotes the Lie derivative along $\boldsymbol{h}: \mathcal{L}_{\boldsymbol{h}} t=h^{\mu} \partial_{\mu} t$. The latter property implies that the 2 -surfaces $\mathcal{S}_{t}$ are Lie dragged by $\boldsymbol{h}$. Let $C$ be half the scalar square of $\boldsymbol{h}$ with respect to the metric $\boldsymbol{g}: \boldsymbol{h} \cdot \boldsymbol{h}=2 C$. It is easy to see that the sign of $C$ gives the signature of the hypersurface $\mathcal{H}: C$ is positive, zero and negative for respectively spacelike, null and timelike hypersurfaces. There exists a unique pair $(\boldsymbol{\ell}, \boldsymbol{k})$ of null vectors normal to $\mathcal{S}_{t}$ and a unique vector $\boldsymbol{m}$ normal to $\mathcal{H}$ such that

$$
\boldsymbol{h}=\boldsymbol{\ell}-C \boldsymbol{k}, \quad \boldsymbol{m}=\boldsymbol{\ell}+C \boldsymbol{k} \quad \text { and } \quad \boldsymbol{\ell} \cdot \boldsymbol{k}=-1
$$

For any vector field $\boldsymbol{v}$ normal to $\mathcal{S}_{t}$, such as $\boldsymbol{h}, \boldsymbol{m}, \boldsymbol{\ell}$ or $\boldsymbol{k}$, we define the expansion $\theta^{(\boldsymbol{v})}$ and the shear tensor $\boldsymbol{\sigma}^{(\boldsymbol{v})}$ of the surface $\mathcal{S}_{t}$ when Lie-dragged along $\boldsymbol{v}$ by

$$
\mathcal{L}_{\boldsymbol{v}} \boldsymbol{q}=\theta^{(\boldsymbol{v})} \boldsymbol{q}+2 \boldsymbol{\sigma}^{(\boldsymbol{v})} \text { and } \operatorname{tr} \boldsymbol{\sigma}^{(\boldsymbol{v})}=0
$$

where $\boldsymbol{q}$ is the induced metric on $\mathcal{S}_{t}$ ( $\boldsymbol{q}$ is positive definite since $\mathcal{S}_{t}$ is assumed to be spacelike), $\mathcal{L}_{\boldsymbol{v}} \boldsymbol{q}$ is its Lie derivative resulting from the dragging of the surface $\mathcal{S}_{t}$ along the normal vector $\boldsymbol{v}$, and $\operatorname{tr} \boldsymbol{\sigma}^{(\boldsymbol{v})}$ is the trace of $\boldsymbol{\sigma}^{(\boldsymbol{v})}$ with respect to the metric $\boldsymbol{q}$. A consequence of (2) is that $\theta^{(\boldsymbol{v})}$ governs the variation of the surface element 2 -form $\boldsymbol{\epsilon}_{\mathcal{S}}$ of $\mathcal{S}_{t}$ according to $\mathcal{L}_{\boldsymbol{v}} \boldsymbol{\epsilon}_{\mathcal{S}}=\theta^{(\boldsymbol{v})} \boldsymbol{\epsilon}_{\mathcal{S}}$.

Let us recall that $\mathcal{S}_{t}$ is called a trapped surface if $\theta^{(\boldsymbol{k})}<$ 0 and $\theta^{(\ell)}<0$, and marginally trapped surface (MTS) if $\theta^{(\boldsymbol{k})}<0$ and $\theta^{(\ell)}=0$. The hypersurface $\mathcal{H}=\bigcup_{t \in \mathbb{R}} \mathcal{S}_{t}$ is said to be a future outer trapping horizon (FOTH) if (i) each $\mathcal{S}_{t}$ is a MTS and (ii) the (local) outermost condition $\mathcal{L}_{k} \theta^{(\ell)}<0$ is satisfied [1]. $\mathcal{H}$ is said to be a dynamical horizon (DH) if (i) each $\mathcal{S}_{t}$ is a MTS and (ii) $\mathcal{H}$ is a spacelike hypersurface 3 . Note that in generic dynamical situations, the notions of FOTH and $\mathrm{DH}$ are equivalent [5]. In stationary situations, a FOTH becomes a null hypersurface, whereas a DH (which by definition 
is spacelike) cannot exist; it should be replaced by the notion of isolated horizon (IH) [4, 5, 10, 11].

If $\mathcal{H}$ is an EH, the 2 -surfaces $\mathcal{S}_{t}$ are not MTS, except in stationary configurations (Kerr black hole). On the contrary they are expanding, by the famous Hawking area increase law [12]: $\theta^{(\ell)}>0$.

Let us denote by $\kappa$ the component along $\boldsymbol{\ell}$ of the "acceleration" of $\boldsymbol{h}$ in the decomposition [13]

$$
\nabla_{h} h=\kappa \ell+\left(C \kappa-\mathcal{L}_{h} C\right) \boldsymbol{k}-\mathcal{D} C
$$

where $\boldsymbol{\nabla}$ is the spacetime connection and $\mathcal{D}$ the connection associated with the metric $\boldsymbol{q}$ in $\mathcal{S}_{t}$. Besides let $\boldsymbol{\Omega}^{(\ell)}$ be the normal fundamental form of the surface $\mathcal{S}_{t}$ (also called connection on the normal bundle) defined by $\boldsymbol{\Omega}^{(\ell)}:=-\boldsymbol{k} \cdot \boldsymbol{\nabla}_{\overrightarrow{\boldsymbol{q}}} \boldsymbol{\ell}$, where $\overrightarrow{\boldsymbol{q}}$ denotes the orthogonal projector on the surface $\mathcal{S}_{t}$ (see e.g. Sec. III.D of Ref. [8]).

From the Einstein equation, one can derive the following evolution law for any foliated hypersurface $\mathcal{H}$ (details are provided in Ref. [13]):

$$
\mathcal{L}_{\boldsymbol{h}} \theta^{(\boldsymbol{m})}=\kappa \theta^{(\boldsymbol{h})}-\frac{1}{2} \theta^{(\boldsymbol{h})} \theta^{(\boldsymbol{m})}-\boldsymbol{\sigma}^{(\boldsymbol{h})}: \boldsymbol{\sigma}^{(\boldsymbol{m})}-8 \pi \boldsymbol{T}(\boldsymbol{m}, \boldsymbol{h})+\theta^{(\boldsymbol{k})} \mathcal{L}_{\boldsymbol{h}} C+\mathcal{D} \cdot\left(2 C \overrightarrow{\boldsymbol{\Omega}}^{(\ell)}-\overrightarrow{\mathcal{D}} C\right),
$$

where $\boldsymbol{T}$ is the energy-momentum tensor of matter (if any), an upper arrow indicates index raising with the metric $\boldsymbol{q}$ and the notation ":" stands for the double contraction, i.e. $\boldsymbol{\sigma}^{(\boldsymbol{h})}: \boldsymbol{\sigma}^{(\boldsymbol{m})}:=\sigma_{a b}^{(\boldsymbol{h})} \sigma^{(\boldsymbol{m}) a b}$. If we specialize Eq. (4) to the cases of (i) an $\mathrm{EH}$ and (ii) a FOTH or a $\mathrm{DH}$, we obtain respectively

$$
\begin{aligned}
\mathcal{L}_{\ell} \theta^{(\ell)}+\left(\theta^{(\ell)}\right)^{2}-\kappa \theta^{(\ell)} & =\frac{1}{2}\left(\theta^{(\ell)}\right)^{2}-\boldsymbol{\sigma}^{(\ell)}: \boldsymbol{\sigma}^{(\ell)}-8 \pi \boldsymbol{T}(\ell, \ell) \\
\mathcal{L}_{\boldsymbol{h}} \theta^{(\boldsymbol{h})}+\left(\theta^{(\boldsymbol{h})}\right)^{2}+\kappa \theta^{(\boldsymbol{h})} & =\frac{1}{2}\left(\theta^{(\boldsymbol{h})}\right)^{2}+\boldsymbol{\sigma}^{(\boldsymbol{h})}: \boldsymbol{\sigma}^{(\boldsymbol{m})}+8 \pi \boldsymbol{T}(\boldsymbol{m}, \boldsymbol{h})-\theta^{(\boldsymbol{k})} \mathcal{L}_{\boldsymbol{h}} C+\mathcal{D} \cdot\left(\overrightarrow{\mathcal{D}} C-2 C \overrightarrow{\boldsymbol{\Omega}}^{(\ell)}\right) .
\end{aligned}
$$

For the $\mathrm{EH}$, we have used the null character of $\mathcal{H}$, which implies $C=0$ and $\boldsymbol{h}=\boldsymbol{m}=\boldsymbol{\ell}$, yielding Eq. (5). This is nothing but the null Raychaudhuri equation for a surfaceorthogonal congruence 14]. In this case Eq. [3) reduces to $\nabla_{\ell} \ell=\kappa \ell$, i.e. $\kappa$ is the non-affinity coefficient of $\boldsymbol{\ell}$ and coincides with the so-called surface gravity for a stationary horizon, provided that $\boldsymbol{\ell}$ is normalized in terms of the stationarity Killing vector. For the FOTH/DH case [Eq. (6)], we have used the property $\theta^{(\ell)}=0$ to write $\theta^{(\boldsymbol{m})}=-\theta^{(\boldsymbol{h})}$, since Eq. (1) gives $\theta^{(\boldsymbol{m})}=-\theta^{(\boldsymbol{h})}+2 \theta^{(\ell)}$.

The area of the 2-surface $\mathcal{S}_{t}$ is $A(t)=\int_{\mathcal{S}_{t}} \boldsymbol{\epsilon}_{\mathcal{S}}$. Since the surfaces $\mathcal{S}_{t}$ are Lie dragged by the vector $\boldsymbol{h}$ associated with $t$, the first and second derivative of the area with respect to $t$ are $d A / d t=\int_{\mathcal{S}_{t}} \theta^{(\boldsymbol{h})} \boldsymbol{\epsilon}_{\mathcal{S}}$ and $d^{2} A / d t^{2}=$ $\int_{\mathcal{S}_{t}}\left[\mathcal{L}_{\boldsymbol{h}} \theta^{(\boldsymbol{h})}+\left(\theta^{(\boldsymbol{h})}\right)^{2}\right] \boldsymbol{\epsilon}_{\mathcal{S}}$. Then, assuming $C>0$ in Eq. (6) (i.e. considering a DH only), we may introduce $\kappa^{\prime}:=-C^{-1} \ell \cdot \nabla_{h} \boldsymbol{h}=\kappa-\mathcal{L}_{\boldsymbol{h}} \ln C$ [cf. Eq. (3)], and integrate Eqs. (5) and (6) over $\mathcal{S}_{t}$, noticing that the integral of the divergence term in Eq. (6) vanishes, to get respectively

$$
\begin{array}{r}
\frac{d^{2} A}{d t^{2}}-\bar{\kappa} \frac{d A}{d t}=-\int_{\mathcal{S}_{t}}\left[8 \pi \boldsymbol{T}(\boldsymbol{\ell}, \boldsymbol{\ell})+\boldsymbol{\sigma}^{(\ell)}: \boldsymbol{\sigma}^{(\ell)}\right. \\
\left.-\frac{\left(\theta^{(\ell)}\right)^{2}}{2}+(\bar{\kappa}-\kappa) \theta^{(\ell)}\right] \boldsymbol{\epsilon}_{\mathcal{S}}, \\
\frac{d^{2} A}{d t^{2}}+\bar{\kappa}^{\prime} \frac{d A}{d t}=\int_{\mathcal{S}_{t}}\left[8 \pi \boldsymbol{T}(\boldsymbol{m}, \boldsymbol{h})+\boldsymbol{\sigma}^{(\boldsymbol{h})}: \boldsymbol{\sigma}^{(\boldsymbol{m})}\right. \\
\left.+\frac{\left(\theta^{(\boldsymbol{h})}\right)^{2}}{2}+\left(\bar{\kappa}^{\prime}-\kappa^{\prime}\right) \theta^{(\boldsymbol{h})}\right] \boldsymbol{\epsilon}_{\mathcal{S}},
\end{array}
$$

where $\bar{\kappa}$ and $\bar{\kappa}^{\prime}$ denote the mean value over $\mathcal{S}_{t}$ of $\kappa$ and $\kappa^{\prime}: \bar{\kappa}=\bar{\kappa}(t):=A^{-1} \int_{\mathcal{S}_{t}} \kappa \epsilon_{\mathcal{S}}$ (idem for $\kappa^{\prime}$ ). Assume for a moment that $\bar{\kappa}$ and $\bar{\kappa}^{\prime}$ are constant and positive, as for the EH of a Kerr black hole with foliations compatible with the stationarity Killing vector. Let us consider first the evolution of an EH, i.e. the differential equation (7). The general solution of the corresponding homogeneous equation is $A(t)=$ const $_{1} \cdot \exp (\bar{\kappa} t)+$ const $_{2}$. Thus, if one were solving Eq. (7) as a Cauchy problem, one would obtain exponentially diverging solutions (since $\bar{\kappa}>0$ ). It is well known that the correct treatment must be teleological ([6, 14] or Sec. VI.C.6 of Ref. [7]), i.e. one imposes the boundary condition $d A / d t=0$ at $t=+\infty$, to get

$$
\frac{d A}{d t}=\int_{t}^{+\infty} D(u) e^{\bar{\kappa}(t-u)} d u
$$

where $D=D(t)$ stands for the integral in the right-hand side of Eq. (7). On the contrary, the differential equation (8) for the DH area is such that the solutions of the homogeneous equation are decaying exponentially. Accordingly, the treatment as a standard Cauchy problem from the initial condition $d A / d t(0)=\dot{A}_{0}$ leads to the non-diverging solution

$$
\frac{d A}{d t}=\dot{A}_{0}+\int_{0}^{t} D^{\prime}(u) e^{\bar{\kappa}^{\prime}(u-t)} d u
$$

where $D^{\prime}=D^{\prime}(t)$ stands for the integral in the righthand side of Eq. (8). The striking difference between Eqs. (9) and (10) is that Eq. (10) is causal (the solution at a given instant $t$ depends only on the behavior of the 
source $D^{\prime}$ at instants $u \leq t$ ), whereas Eq. (9) is not. This reflects the non-local character of EHs mentioned in the Introduction. Of course, in general $\bar{\kappa}$ and $\bar{\kappa}^{\prime}$ are not constant (except for small perturbations of a stationary black hole [15]), but the behavior described above should remain the same.

Comparing with previous works, one can show that at the limit of small departure from an IH, the area law (8) reduces to that established for slowly evolving horizons by Booth \& Fairhurst 15. In the full dynamical regime, the evolution law (8) is different from the area law obtained by Ashtekar \& Krishnan [3, 4] (see also 16]). Indeed the latter is derived from a different component of Einstein equation: the $\boldsymbol{T}(\boldsymbol{m}, \boldsymbol{\ell})$ one instead of $\boldsymbol{T}(\boldsymbol{m}, \boldsymbol{h})$ for Eq. (8). More precisely, the Ashtekar-Krishnan law is written in terms of the areal radius $R:=\sqrt{A / 4 \pi}$ and expresses the variation of $R$ between two surfaces $\mathcal{S}_{t_{1}}$ and $\mathcal{S}_{t_{2}}$ (cf. Eq. (3.25) of Ref. [3]). Using our notations (cf. Table II of Ref. [8] for the correspondence) its differential version (i.e. writing $t_{2}=t_{1}+d t$ ) is

$$
\begin{aligned}
\frac{1}{2} \frac{d R}{d t}= & \frac{1}{8 \pi} \int_{\mathcal{S}_{t}}\left[\boldsymbol{\Omega}^{(\tilde{\ell})} \cdot \overrightarrow{\boldsymbol{\Omega}}^{(\tilde{\ell})}+\frac{1}{C} \boldsymbol{\sigma}^{(\ell)}: \boldsymbol{\sigma}^{(\ell)}\right] \frac{d R}{d t} \boldsymbol{\epsilon}_{\mathcal{S}} \\
& +\int_{\mathcal{S}_{t}} \frac{1}{C} \boldsymbol{T}(\boldsymbol{m}, \boldsymbol{\ell}) \frac{d R}{d t} \boldsymbol{\epsilon}_{\mathcal{S}},
\end{aligned}
$$

where $\boldsymbol{\Omega}^{(\tilde{\ell})}:=\boldsymbol{\Omega}^{(\ell)}-\mathcal{D} \ln C$. Notice that this is a first order equation in $A(t)=4 \pi R(t)^{2}$, whereas Eq. (8) is of second order. Moreover, it contains $d R / d t$ on both sides, so that one can divide by $d R / d t$ to get an equation which does not contain $R(t)$. In this respect, Eq. (11) does not appear as an evolution equation for $A(t)$. Actually, as shown by Hayward 2], Eq. (11) can be obtained by integrating over $\mathcal{S}_{t}$ the relation expressing that $\mathcal{L}_{\boldsymbol{h}} \theta^{(\ell)}=0$ on $\mathcal{H}$. This relation involves $\boldsymbol{T}(\boldsymbol{m}, \boldsymbol{\ell})$, in the same manner as Eq. (4) relates $\mathcal{L}_{\boldsymbol{h}} \theta^{(\boldsymbol{m})}$ and $\boldsymbol{T}(\boldsymbol{m}, \boldsymbol{h})$. Explicitly we have [2, 13.

$$
\begin{gathered}
C\left[\mathcal{D} \cdot \overrightarrow{\boldsymbol{\Omega}}^{(\tilde{\ell})}+\boldsymbol{\Omega}^{(\tilde{\ell})} \cdot \overrightarrow{\boldsymbol{\Omega}}^{(\tilde{\ell})}-\frac{1}{2} \mathcal{R}\right]+\boldsymbol{\sigma}^{(\ell)}: \boldsymbol{\sigma}^{(\ell)} \\
+8 \pi \boldsymbol{T}(\boldsymbol{m}, \boldsymbol{\ell})=0
\end{gathered}
$$

where $\mathcal{R}$ denotes the Ricci scalar associated with the metric $\boldsymbol{q}$ in $\mathcal{S}_{t}$. Dividing Eq. (12) by $8 \pi C$, integrating over $\mathcal{S}_{t}$, invoking the Gauss-Bonnet theorem to set the integral of $\mathcal{R}$ to $8 \pi$ and multiplying by $d R / d t$ leads to the differential form (11) of Ashtekar-Krishnan law.

Energy dissipation and bulk viscosity. In the membrane paradigm approach to black holes, Price and Thorne [7] defined the surface energy density of an EH as $\varepsilon:=$ $-\theta^{(\ell)} / 8 \pi$ and interpreted Eq. (5) as an energy balance law, with heat production resulting from viscous stresses. By analogy, let us define the surface energy density of a FOTH/DH as $\varepsilon:=-\theta^{(\boldsymbol{m})} / 8 \pi$, where the role of the normal to $\mathcal{H}$ is now taken by $\boldsymbol{m}$ instead of $\boldsymbol{\ell}$. Since $\theta^{(\boldsymbol{m})}=-\theta^{(\boldsymbol{h})}$ for a FOTH/DH, we have $\varepsilon=\theta^{(\boldsymbol{h})} / 8 \pi$ and we may rewrite Eq. (6) as

$$
\mathcal{L}_{\boldsymbol{h}} \varepsilon+\theta^{(\boldsymbol{h})} \varepsilon=-\frac{\kappa}{8 \pi} \theta^{(\boldsymbol{h})}+\frac{1}{8 \pi} \boldsymbol{\sigma}^{(\boldsymbol{h})}: \boldsymbol{\sigma}^{(\boldsymbol{m})}+\frac{\left(\theta^{(\boldsymbol{h})}\right)^{2}}{16 \pi}
$$

$$
-\mathcal{D} \cdot \boldsymbol{Q}+\boldsymbol{T}(\boldsymbol{m}, \boldsymbol{h})-\frac{\theta^{(\boldsymbol{k})}}{8 \pi} \mathcal{L}_{\boldsymbol{h}} C,
$$

with $\boldsymbol{Q}:=\frac{1}{4 \pi}\left[C \overrightarrow{\boldsymbol{\Omega}}^{(\ell)}-1 / 2 \overrightarrow{\mathcal{D}} C\right]=-\frac{C}{4 \pi} \vec{\varpi}$, where $\varpi$ is the anholonomicity 1-form (or twist 1-form) of the 2 -surface $\mathcal{S}_{t}$ [1] (see also Sec. IV.A of Ref. [8]) and $\vec{\varpi}$ denotes its vector dual. It is worth to write aside Eq. (13) the generalized Damour-Navier-Stokes equation [8]

$$
\begin{aligned}
\mathcal{L}_{h} \boldsymbol{\pi}+\theta^{(\boldsymbol{h})} \boldsymbol{\pi}= & -\mathcal{D}\left(\frac{\kappa}{8 \pi}\right)+\mathcal{D} \cdot\left(\frac{\overrightarrow{\boldsymbol{\sigma}}^{(\boldsymbol{m})}}{8 \pi}\right)+\frac{\mathcal{D} \theta^{(\boldsymbol{h})}}{16 \pi} \\
& -\boldsymbol{T}(\boldsymbol{m}, \overrightarrow{\boldsymbol{q}})+\frac{\theta^{(\boldsymbol{k})}}{8 \pi} \mathcal{D} C,
\end{aligned}
$$

where $\boldsymbol{\pi}:=-\boldsymbol{\Omega}^{\ell} / 8 \pi$. It is striking that Eqs. (13)(14) are fully analogous to the equations that govern a two-dimensional non-relativistic fluid of internal energy density $\varepsilon$, momentum density $\boldsymbol{\pi}$, pressure $\kappa / 8 \pi$, shear stress tensor $\boldsymbol{\sigma}^{(\boldsymbol{m})} / 8 \pi$, bulk viscosity $\zeta=1 / 16 \pi$, shear strain tensor $\boldsymbol{\sigma}^{(\boldsymbol{h})}$, expansion $\theta^{(\boldsymbol{h})}$, subject to the external force density $-\boldsymbol{T}(\boldsymbol{m}, \overrightarrow{\boldsymbol{q}})+\theta^{(\boldsymbol{k})} / 8 \pi \mathcal{D} C$, external energy production rate $\boldsymbol{T}(\boldsymbol{m}, \boldsymbol{h})-\theta^{(\boldsymbol{k})} / 8 \pi \mathcal{L}_{\boldsymbol{h}} C$ and heat flux $Q$ (see e.g. Ref. [17]). Let us notice that the shear viscosity $\mu$ does not appear in Eqs. (13)-(14), because the standard Newtonian-fluid relation between the shear stress tensor $\boldsymbol{\sigma}^{(\boldsymbol{m})} / 8 \pi$ and the shear strain tensor $\boldsymbol{\sigma}^{(\boldsymbol{h})}$, namely $\boldsymbol{\sigma}^{(\boldsymbol{m})} / 8 \pi=2 \mu \boldsymbol{\sigma}^{(\boldsymbol{h})}$, does not hold. Here we have $\boldsymbol{\sigma}^{(\boldsymbol{m})} / 8 \pi=\left[\boldsymbol{\sigma}^{(\boldsymbol{h})}+2 C \boldsymbol{\sigma}^{(\boldsymbol{k})}\right] / 8 \pi$, so that the Newtonian-fluid assumption is fulfilled only if $C=0$ (IH limit). On the contrary, it appears from Eqs. (13)(14) that the trace part of the viscous stress tensor $\boldsymbol{S}_{\text {visc }}$ obeys the Newtonian-fluid law, being proportional to the trace part of the strain tensor (i.e. the expansion $\theta^{(\boldsymbol{h})}$ ): $\operatorname{tr} \boldsymbol{S}_{\text {visc }}=3 \zeta \theta^{(\boldsymbol{h})}$.

Let us point out two differences with the EH case [6, 7]. First the heat flux $\boldsymbol{Q}$ is not vanishing for a FOTH/DH, whereas it was zero for an EH. Notice that $\boldsymbol{Q}$ is a vector tangent to $\mathcal{S}_{t}$ so that the integration of Eq. (13) over the closed surface $\mathcal{S}_{t}$ to get a global internal energy balance law would not contain any net heat flux. The second major difference is that the bulk viscosity $\zeta$ is positive, being equal to $1 / 16 \pi$, whereas it was found to be negative, being equal to $-1 / 16 \pi$, for EHs [6, 7]. This negative value, which would yield to a dilation or contraction instability in an ordinary fluid, is in agreement with the tendency of a null hypersurface to continually contract or expand, the EH being stabilized by the teleological condition imposing its expansion to vanish in the far future. The positive value of the bulk viscosity found here shows that FOTHs and DHs behave as "ordinary" physical objects and is in perfect agreement with their local nature.

Entropy evolution and choice of $\mathcal{H}$. Hitherto we have discussed the horizon $\mathcal{H}$ as a given hypersurface in $\mathcal{M}$. We adopt now a $3+1$ approach and consider, in the line of Ref. [18], the world-tube evolution of a MTS $\mathcal{S}_{0}$ contained in an initial Cauchy slice $\Sigma_{0}$ of a $3+1$ foliation $\left(\Sigma_{t}\right)_{t \in \mathbb{R}}$. Denoting by $s$ the unit-normal vector to $\mathcal{S}_{0}$ contained in $\Sigma_{0}$ and by $\boldsymbol{n}$ the unit-normal vector to $\Sigma_{0}$, we decompose 
$\mathcal{H}$ 's "time evolution" vector as $\boldsymbol{h}=N \boldsymbol{n}+b \boldsymbol{s}$, where $N$ is the lapse function associated with the time parameter $t$ of the $3+1$ foliation. Introducing the null vectors $\hat{\boldsymbol{\ell}}:=\boldsymbol{n}+\boldsymbol{s}$ and $\hat{\boldsymbol{k}}:=(\boldsymbol{n}-\boldsymbol{s}) / 2$, we get $\boldsymbol{h}=(b+N) / 2 \hat{\boldsymbol{\ell}}+(b-N) \hat{\boldsymbol{k}}$ and $C=\left(b^{2}-N^{2}\right) / 2$. Combined uniqueness results from Refs. 9, 18 show that: (i) to each $3+1$ foliation $\left(\Sigma_{t}\right)_{t \in \mathbb{R}}$ there corresponds a unique $\mathrm{DH}$ containing $\mathcal{S}_{0}$ and sliced by MTSs $\mathcal{S}_{t} \subset \Sigma_{t}$; and (ii) different $3+1$ slicings lead generically to different $\mathrm{DH}$. In other words, the evolution of $\mathcal{S}_{0}$ into MTSs is an ill-defined concept, since different DHs pass through $\mathcal{S}_{0}$. A natural question consists in introducing a notion of preferred DH or, equivalently, of a preferred $3+1$ slicing.

With this ultimate aim, let us push forward the viscous fluid interpretation of FOTH/DHs. As an ever growing quantity on $\mathcal{H}$, it is natural to interpret the area $A$ as proportional to the entropy of the horizon, the equilibrium case providing the relation $S=\frac{k_{B}}{4 \hbar} A$ [1, 3, 15]. In this sense, Eq. (5) for an EH has been interpreted in the membrane paradigm [6, 7] as a viscous fluid entropy dissipation equation for the differential element of entropy, with the specificity of involving a second time derivative term in the entropy production. Instead of the analogous discussion for FOTH/DHs based on Eq. (6), we take the condition $\mathcal{L}_{\boldsymbol{h}} \theta^{(\ell)}=0$, i.e. Eq. (12), as a starting point. When expressed in terms of $3+1$ quantities, it becomes an elliptic equation on $(b-N)$ with a source proportional to $(b+N)$ [13, 19]. In this $3+1$ approach, a second relation between $(b-N)$ and $(b+N)$ is necessary in order to determine $\mathcal{H}$.

In a first attempt to get this second relation, we consider a maximum entropy production criterion, which can be motivated in the context of non-equilibrium thermodynamics [20]. Maximizing $d S / d t$ leads to [13]

$$
b-N=-\operatorname{const} \cdot \theta^{(\hat{\boldsymbol{k}})} \text {. }
$$

In loose terms, and independently of thermodynamic con- siderations, Eq. (15) singles out the DH that approaches "the fastest" to the EH. However it leads to a function $A(t)$ which is only $\mathcal{C}^{0}$ in the matching with an initial IH.

Alternatively, we can control the response of $\mathcal{H}$ to the arrival of energy/matter, by prescribing the convexity of the entropy in time. We propose a phenomenological choice for the second derivative of the area element $\ddot{a}:=$ $\mathcal{L}_{\boldsymbol{h}} \theta^{(\boldsymbol{h})}+\left(\theta^{(\boldsymbol{h})}\right)^{2}$ :

$$
\ddot{a}=F[\text { sources }]+\alpha \frac{\theta^{(\boldsymbol{h})}}{C} \mathcal{L}_{\boldsymbol{h}} C+\beta \frac{\theta^{(\boldsymbol{h})}}{C} \mathcal{L}_{\boldsymbol{h}} \mathcal{L}_{\boldsymbol{h}} C,
$$

such that $F$ [sources] $>0$ whenever matter or gravitational radiation crosses the horizon $[$ e.g. $\boldsymbol{T}(\boldsymbol{\ell}, \boldsymbol{\ell}) \neq 0$ or $\boldsymbol{\sigma}^{(\ell)} \neq 0$, and $F$ [sources] $\leq 0$ otherwise. Inserting Eq. (16) into Eq. (6) leads to a second-order evolution equation for $C=\left(b^{2}-N^{2}\right) / 2$, whose resolution provides the additional relation between $(b-N)$ and $(b+N)$. Initial condition $C=0$ guarantees the $\mathcal{C}^{1}$ matching with an IH, whereas the choice of initial $\mathcal{L}_{\boldsymbol{h}} C$ amounts to the choice of $\mathrm{DH}$ (consistency requires an appropriate choice of parameters $\alpha, \beta)$. Alternatively, the equation for $C$ can be seen as a (now first-order) balance equation for the element of entropy. Interpreting it as a non-equilibrium thermodynamics Clausius-Duhem-like inequality [20] (by enforcing the positivity of the entropy production), provides a guideline for fixing the phenomenological term $F$ [sources]. Other phenomenological options to Eq. (16) can be proposed, and we aim at exploring them numerically [13]. A tempting possibility is to base the (unavoidable) choice of $\mathrm{DH}$, upon an entropy principle derived solely from the structure of the hyperbolic system defined by (part of ) the Einstein equations on $\mathcal{H}$.

Acknowledgements. We thank J. Metzger and L. Andersson for the discussion on the maximization on the area change. JLJ acknowledges the support of the Marie Curie Intra-European contract MEIF-CT-2003-500885 within the 6th European Community Framework Programme.
[1] S.A. Hayward, Phys. Rev. D 49, 6467 (1994).

[2] S.A. Hayward, Phys. Rev. Lett. 93, 251101 (2004); Phys. Rev. D 70, 104027 (2004).

[3] A. Ashtekar and B. Krishnan, Phys. Rev. D 68, 104030 (2003).

[4] A. Ashtekar and B. Krishnan, Living Rev. Relativity 7, 10 (2004) [Online article]: cited on 21 June 2006, http://www. livingreviews.org/lrr-2004-10

[5] I. Booth, Can. J. Phys. 83, 1073 (2005).

[6] T. Damour : Quelques propriétés mécaniques, électromagnétiques, thermodynamiques et quantiques des trous noirs, Thèse de doctorat d'État, Université Paris 6 (1979); in Proceedings of the Second Marcel Grossmann Meeting on General Relativity, Ed. R. Ruffini, North Holland (1982), p. 587.

[7] K.S. Thorne, R.H. Price and D.A. MacDonald : Black holes : the membrane paradigm, Yale University Press, New Haven (1986); R.H. Price and K.S. Thorne, Phys.
Rev. D 33, 915 (1986).

[8] E. Gourgoulhon, Phys. Rev. D 72, 104007 (2005).

[9] A. Ashtekar and G.J. Galloway, Adv. Theor. Math. Phys. 9, 1 (2005).

[10] A. Ashtekar, C. Beetle, and S. Fairhurst, Class. Quantum Grav. 16, L1 (1999).

[11] E. Gourgoulhon and J.L. Jaramillo, Phys. Rep. 423, 159 (2006).

[12] S.W. Hawking, Commun. Math. Phys. 25, 162 (1972).

[13] E. Gourgoulhon and J.L. Jaramillo, in preparation.

[14] S.W. Hawking and J.B. Hartle, Commun. Math. Phys. 27, 283 (1972).

[15] I. Booth and S. Fairhurst, Phys. Rev. Lett. 92, 011102 (2004).

[16] S.A. Hayward, preprint gr-qc/0607081

[17] M. Rieutord : Une introduction à la dynamique des fluides, Masson, Paris (1997).

[18] L. Andersson, M. Mars, and W. Simon, Phys. Rev. Lett. 
95111102 (2005).

[19] D.M. Eardley, Phys. Rev. D 57, 2299 (1998).

[20] A. Greven, G. Keller, and G. Warnecke : Entropy,
Princeton Univ. Press, Princeton (2003). 